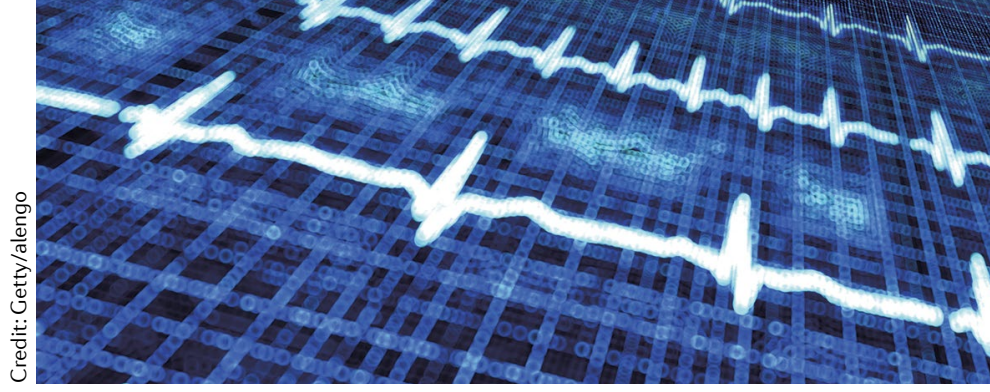

ARTIFICIAL INTELLIGENCE

\section{Artificial intelligence to improve the diagnosis of cardiovascular diseases}

Deep-learning algorithms applied to a widely available and low-cost test, the electrocardiogram (ECG), can improve the identification and classification of cardiac arrhythmias and the detection of early-stage heart disease, according to two studies published in Nature Medicine. "Automating arrhythmia detection can make heart monitoring with ECG more accessible and useful as a first-line diagnostic tool," says Awni Hannun, corresponding author of one of the articles. "These results have the potential to lead to reduced rates of currently misdiagnosed, computerized ECG interpretations, and to improvements in efficiency of expert-human ECG interpretation."

Hannun and colleagues used a large training dataset including 91,232 single-lead ECG records from 53,549 patients to develop a deep neural network to classify 10 arrhythmias, sinus rhythm and noise for a total of 12 output rhythm classes. "In the past, most work has focused on only a few of the sometimes easier to diagnose arrhythmias such as atrial fibrillation, but in this work we diagnose a much larger set including various forms of heart block," explains Hannun. The researchers then validated the deep neural network on an independent test dataset (328 ECG records from 328 patients) that had been annotated by a consensus committee of board-certified cardiologists. Each ECG record in the test dataset was also classified by six cardiologists who were not part of the committee. The deep neural network matched the performance of board-certified cardiologists - the algorithm had an average area under the receiver operating characteristic curve (AUC), in which 1.00 would be a perfect classification and 0.50 a random classification, of 0.97 - and exceeded the average cardiologist sensitivity for all rhythm classes, with a higher average $F_{1}$ score (the harmonic mean of the positive predictive value and sensitivity) than cardiologists ( 0.837 versus 0.780 ).

In another study, Attia et al. used artificial intelligence applied to the ECG for the detection of asymptomatic left ventricular (LV) dysfunction, a precursor of heart failure. The team used paired 12-lead ECG and echocardiogram data from 44,959 patients to train a deep-learning approach, known as a convolutional neural network, to identify patients with LV dysfunction (ejection fraction <35\%) using only the ECG record. The network model was then validated on an independent dataset of 52,870 patients. The model had an AUC of 0.93 for diagnosis of mechanical dysfunction, and a sensitivity, specificity, accuracy and $\mathrm{F}_{1}$ score of $86.3 \%, 85.7 \%, 85.7 \%$ and $49.5 \%$, respectively. Of note, 1,335 patients without LV dysfunction were assigned by the algorithm as having low ejection fraction. Attia et al. suggest that these 'false positives' are an early detection of ECG abnormalities, given that the risk of developing future LV dysfunction was higher in patients with an initial 'false positive' diagnosis than in those with a negative diagnosis (HR 4.1, 95\% CI 3.3-5.0). The 5-year incidence was $9.5 \%$.

These studies demonstrate the potential of deep-learning approaches to improve the accuracy and efficacy of ECG reading for patient screening and stratification.

Irene Fernández-Ruiz

ORIGINAL ARTICLE Hannun, A. Y. et al. classification in ambulatory electrocardiograms using a deep neural network. Nat. Med. 25, 65-69 (2019) | Attia, Z. et al. Screening for cardiac contractile dysfunction using an artificial intelligence-enabled electrocardiogram. Nat. Med. 25, 70-74 (2019) of LV dysfunction in these patients Cardiologist-level arrhythmia detection and

\section{RISK FACTORS}

\section{Income volatility and increased risk of CVD}

Volatility in income is associated with an increased risk of cardiovascular disease (CVD) and higher all-cause mortality, according to data from the Coronary Artery Risk Development in Young Adults Study. This ongoing, prospective cohort study is being conducted in four cities in the USA: Birmingham, Alabama; Chicago, Illinois; Minneapolis, Minnesota and Oakland, California. Income volatility (defined as the intra-individual s.d. of the percentage change in income across five assessments from 1990 to 2005) was associated with increased risk of CVD (HR 2.07, 95\% CI 1.10-3.90) and all-cause mortality (HR 1.78, 95\% Cl 1.03-3.09). Similarly, a greater number of income drops (defined as a decrease of $\geq 25 \%$ from the previous visit and less than the participant's average income from 1990 to 2005) was also associated with increased risk of CVD (HR 2.54, 95\% Cl 1.24-5.19) and all-cause mortality (HR 1.92, 95\% Cl 1.07-3.44). "The findings of this study reinforce the urgency and growing public health threat associated with income volatility in the United States," conclude the investigators.

ORIGINAL ARTICLE Elfassy, T. et al. Association of income volatility with incident cardiovascular disease and all-cause mortality in a US cohort: 1990 to 2015. Circulation https://doi.org/10.1161/CIRCULATIONAHA.118.035521 (2019)

\section{$\Rightarrow$ ANTITHROMBOTIC THERAPY}

\section{Adding rivaroxaban to aspirin after CABG surgery}

In the COMPASS trial, the combination of rivaroxaban and aspirin reduced the rate of major adverse cardiovascular events (MACE) compared with aspirin alone in individuals with stable atherosclerotic vascular disease. In a pre-planned substudy (the COMPASS-CABG study), the regimens of aspirin alone, $5.0 \mathrm{mg}$ of rivaroxaban alone or aspirin plus $2.5 \mathrm{mg}$ of rivaroxaban were compared in 1,448 patients from the COMPASS trial undergoing CABG surgery. Neither rivaroxaban alone nor the combination of aspirin plus rivaroxaban reduced the rate of graft failure (the primary outcome) compared with aspirin alone. The combination therapy was associated with a nonsignificant reduction in the rate of MACE (HR 0.69, 95\% $\mathrm{Cl} 0.33-1.47, P=0.34$ ), whereas rivaroxaban alone was not.

ORIGINAL ARTICLE Lamy, A. et al. Rivaroxaban, aspirin, or both to prevent early coronary bypass graft occlusion: the COMPASS-CABG study.J. Am. Coll. Cardiol. 73, 121-130 (2019)

\section{$\Rightarrow$ ISCHAEMIA}

\section{Ketone body utilization in myocardial ischaemia}

Ketone bodies, such as acetoacetate and $\beta$-hydroxybutyrate, are metabolites that are alternative sources of energy to glucose and fatty acids, and utilization of ketones has been shown to be upregulated in diabetes mellitus. A retrospective study performed at a single centre in Japan now indicates that ketone body utilization is reduced under conditions of myocardial ischaemia. Coronary and systemic serum lactate and $\beta$-hydroxybutyrate concentrations were measured in 171 consecutive patients with angina who were undergoing an acetylcholine provocation test at Kumamoto University Hospital, Japan. The investigators found that acute coronary lactate production (indicative of increased myocardial anaerobic metabolism) was associated with decreased myocardial $\beta$-hydroxybutyrate consumption, particularly in male patients. Further research into the effects of mild ketosis on cardiovascular prognosis is needed.

ORIGINAL ARTICLE Arima, Y. et al. Myocardial ischemia suppresses ketone body utilization.J. Am. Coll. Cardiol. 73, 246-247 (2019) 